\title{
Appréciation de l'état de santé et Prévalence des parasites internes et de la Peste-Des-Petits-Ruminants chez les ovins dans la Commune Rurale de Thiou (Yatenga) au Burkina Faso
}

Salimata POUSGA ${ }^{\left({ }^{* 1}\right)}$, Boukary NANA $^{(2)}$, Vinsoun MILLOGO $^{(1)}$, Hassane Bismark NACRO $^{(1)}$

${ }^{1}$ Institut du Développement Rural, Université NAZI BONI, BP 1091, Bobo-Dioulasso, Burkina Faso.

${ }^{2}$ Ministère des Ressources Animales, Ecole National d'Elevage et de Santé Animale (ENESA), BP 7026, Ouagadougou, Burkina Faso.

Auteur correspondant: *pousgasalimata@yahoo.fr, Téléphone:(+226)71092576

Mots clés: Santé, Parasites internes, Peste des petits Ruminants, Province du Yatenga

Key words: Health, Internal parasites, Peste-Des-Petits-Ruminants, Yatenga

Publication date 31/08/2019, http://www.m.elewa.org/JAPS

\section{RÉSUMÉ}

Le but de cette étude était de faire la recherche-diagnostique de certaines pathologies ovines. Pour cela, une enquête a été réalisée auprès de 100 éleveurs répartis dans 5 villages, suivis de prélèvement de fèces et de sang dans trois villages pour la coproscopie et la sérologie. Au total 150 prélèvements de sang et de fèces ont été analysés. Les problèmes cutanés, les lésions de pattes et les diarrhées ont étés les principaux symptômes de maladies décrits par les éleveurs. Une prévalence d'infestation parasitaire moyenne de $86,7 \%$ et un nombre moyen d'Euf Par Gramme de fèces de $1149 \pm 706$ ont été trouvés. Des prévalences de $31,6 \%, 85,0 \%$ et 3,33 \%, ont été trouvées respectivement pour les Nématodes, les Coccidies et les Cestodes $(\mathrm{P}<0,05)$. La prévalence était de $44,2 \%$ pour les âges compris entre16 et 24 mois et de 7,70\% pour les âges inferieurs à 15 mois $(P<0,05)$. La prévalence des infestations a été plus élevée dans le village de Nomon $(36,5 \%)$ contre 30,7 et 32,7\% pour les villages de Nodin et Thiou. Une séroprévalence de 36,7\% a été trouvé pour la PesteDes -Petits Ruminants. La prévalence était plus élevée chez les ovins âgés de plus de 25 mois $(45,5 \%)(P<0,05)$, et le village de Nodin à présenté une séroprévalence plus élevée $(59,1 \%)$ comparativement à Thiou $(22,7 \%)$ et Nomon $(18,2 \%)(P<0,05)$. Il a été conclu que les coccidies, les helminthes et la Peste-Des-Petits Ruminants constituent toujours des menaces chez les ovins à Thiou.

Health Status Assessment, Prevalence of internal parasites and Peste-Des-Petits-Ruminants in sheep farms in the Rural Community of Thiou (Yatenga) in Burkina Faso

\footnotetext{
ABSTRACT

The purpose of this study was to determine the prevalence of some important sheep diseases in Yatenga province of Burkina Faso. Therefore, a survey was conducted among 100 farmers in five villages, followed by faeces and blood sampling in three villages for coproscopy and serology. A total of 150 blood and faeces samples were analysed. The survey reported that skin and leg problems in addition to diarrhoea were the main disease symptoms described by the farmers. The laboratory data showed average internal parasite
} 
prevalence of $86.7 \%$ and average Oocysts per Faeces Gramm of $1149 \pm 706$. Prevalence of $31.6 \%, 85.0 \%$ and $3.33 \%$ were found for Nematodes, Coccidia and Cestodes respectively ( $P$ $<0.05$ ), and prevalence were $76.9 \%$ for females and $23.0 \%$ for males. Average OPG numbers were higher in males $(2250 \pm 902)$ compared to females $(819 \pm 259)(P<0.05)$. Parasite prevalence were $44.2 \%$ for sheep with ages ranging between 16 and 24 months, and 7.70\% for those less than 15 months old $(P<0.05)$. Overall parasite prevalence was higher in the village of Nomon (36.5\%) against 30.7 and $32.7 \%$ for the villages of Nodin and Thiou. A seroprevalence of $36.7 \%$ was found for Peste-Des-Petits -Ruminants with a variation by sex showing $8.80 \%$ in males and $43.8 \%$ in females $(P<0.05)$. The prevalence was higher in sheep older than 25 months compared to the other age groups $(45.5 \%)(P<0.05)$. Higher seroprevalence was observed in Nodin $(59.1 \%)$ compared to Thiou $(22.7 \%)$ and Nomon villages $(18.2 \%)(P<0.05)$. It was concluded that Coccidia, Helminths and Peste-Des-Petits -Ruminants are still threats against sheep production in Thiou.

\section{INTRODUCTION}

L'Agriculture burkinabè emploie plus de $86 \%$ de la population totale et contribue pour environ $40 \%$ au PIB du pays dont 25\% pour les productions agricoles, $14 \%$ pour les productions animales et $3 \%$ pour les productions forestières. Ainsi, ses trois domaines constituent les principales sources de croissance économique du pays (MRA, 2004). L'élevage des Petits Ruminants est pratiqué par la plupart des communautés et des ménages ruraux auprès desquels il se présente comme une alternative à la lutte contre la pauvreté (Kabore, 2009). La conduite des Petits Ruminants est facile car ils sont simple à manipuler et peu exigeants en qualité de fourrage (Tchouamo et al., 2005). Cependant, les pathologies constituent l'un des obstacles les plus importants à l'amélioration de la productivité de ces animaux en Afrique (Dagnachew et al., 2011). C'est ainsi que les helminthoses ont été identifiées comme un des facteurs limitant à l'expression du potentiel zootechnique (viande et lait) des animaux (Wesongah et al., 2005 ; Opara, 2005). La PesteDes-Petits-Ruminants (PPR) comme biens d'autres pathologies demeurent toujours la grande hantise des éleveurs (Couacy-Hymann, 2007 ; Sow et al., 2008 ; Kamissoko et al., 2013). L'importance actuelle de ces maladies animales, leur émergence ou réémergence montrent que la surveillance est indispensable pour que soient définies des mesures de lutte adaptées.

Conscients du rôle des Petits Ruminants dans les processus de sécurité alimentaire et nutritionnelle en milieu rural, mais aussi de leur importance dans les systèmes intégrés agriculture-élevage, des innovations sont entreprises par des partenaires du développement rural (ONG, Projets services de vulgarisation) en vue d'accroitre leur productivité. C'est dans cette vision que le projet pour l'Intensification durable des Systèmes Intégrés Agriculture-Elevage (ISIAE) a été élaboré par l'Association pour la Promotion de l'Elevage en Savane et au Sahel (APESS), en vue d'accroitre la productivité agropastorale et la sécurité alimentaire en Afrique de l'Ouest et du Centre. Le présent travail s'inscrit dans le cadre de l'un des objectifs du projet, qui consistait à proposer des paquets technologiques de santé animale adaptés dans la commune rurale de Thiou dans la province du Yatenga qui est aussi une importante zone d'élevage du Burkina Faso. Cependant, le développement de paquet technologique en santé nécessite tout d'abord la connaissance du type de pathologies qui prévalent dans la zone concernée afin de pouvoir dresser un programme de lutte adéquat d'où l'objectif de la présente étude. 


\section{MATÉRIEL ET MÉTHODES}

3.1 Site de l'étude : L'étude s'est déroulée dans la province du Yatenga, Chef-lieu Ouahigouya. La province du Yatenga est située dans la Région du Nord (entre les latitudes $13^{\circ}$ 06 et $14^{\circ} 26$ Nord, et les longitudes $1^{\circ}$ et $3^{\circ}$ Ouest). Le climat est de type soudano-sahélien caractérisé par deux saisons fortement contrastées : la saison sèche et la saison des pluies. La saison sèche d'octobre à mai comporte une période froide $\left(14^{\circ} \mathrm{C}\right.$ à $\left.36^{\circ} \mathrm{C}\right)$ d'octobre à février. Quant à la saison des pluies, elle s'étale sur une courte période (juillet à octobre). La pluviométrie connait depuis une dizaine d'années une forte variabilité $(480 \mathrm{~mm}$ à $813 \mathrm{~mm}$ par an) et la moyenne annuelle se situe autour de $650 \mathrm{~mm}$ (Marchal, 1983). La province $\mathrm{du}$ Yatenga appartient au secteur phytogéographique sub-sahelien (Fontes et Guinko, 1995). Une végétation de type arboré est rencontrée dans les bas-fonds avec des espèces comme Vitellaria paradoxa, Parkia biglobosa. L'activité principale dans cette zone est la production céréalière $(90 \%$ des terres cultivée) à base de mil et de sorgho. L'élevage occupe une place importante dans l'économie des exploitations après l'agriculture, et l'élevage de petits ruminants constitue une source de revenu régulier après la volaille.

3.2 Critères de sélection des élevages : Le choix des producteurs a été fait selon leurs expériences antérieures dans les activités de l'Association pour la Promotion de l'Elevage au Sahel et en Savane (APESS), selon la disponibilité du producteur à participer à l'étude durant toute la période concernée. Ainsi, cinq (5) villages ont été sélectionnés et dix (10) élevages par village ont été enquêtés. Les villages concernés étaient ceux de Thiou, Nodin, Soro, Nomon et Senokayel.

3.3 Collecte des données : La collecte des données s'est faite en deux étapes: une première étape basée sur des enquêtes auprèsdes producteurs et une seconde étape basée sur la collecte des prélèvements de sang et de fèces. Un effectif de 50 élevages a été enquêté avec deux individus questionnés dans chaque élevage, ce qui faisait un total de 100 personnes enquêtées. Cent cinquante (150) prélèvements de sang et de fèces ont été réalisés au total (sur 5 sujets au niveau de chaque élevage) dans les villages de Thiou, Nomon et Nodin.

3.3.1 Les questionnaires : Le questionnaire adressé aux éleveurs comprenait des questions sur les caractéristiques socio-économiques, les principales maladies ovines fréquemment rencontrées, les contraintes liées à l'élevage des ovins ainsi que des questions sur les perceptions générales concernant le traitement des pathologies ovines.

3.3.2 Les enquête : Les entretiens avaient été réalisés en Mooré (une langue nationale burkinabé) par un étudiant en stage qui parlait couramment cette langue. Les questionnaires avaient été vérifiés pour leur exactitude immédiatement après les interviews.

3.3.3 Déclaration d'éthique: Tous les ménages participants avaient été informés de l'objectif de l'étude et du fait que leur participation était volontaire et anonyme. Le consentement verbal avait été obtenu de chaque ménage et documenté dans le questionnaire. Avant l'étude, les membres de l'équipe du projet avaient organisés une mission dans la province du Yatenga pour discuter avec les membres des Comité Villageois pour le Développement (CVD) de la commune rurale pour faciliter le choix des villages et des ménages.

3.3.4 Prélèvements de matières fécales et du sang: Les collectes de matières fécales et du sang ont été réalisées sur 150 ovins. Les fèces ont été prélevés manuellement dans le rectum de chaque animal à l'aide des gants en latex recouverts de poches plastiques, puis transférés dans une glacière et conservés au réfrigérateur à une température de $4^{\circ} \mathrm{C}$. Les prélèvements de sang ont été effectués au niveau de la veine jugulaire dans des tubes de 4 millilitres ( $\mathrm{ml}$ ) sous vide avec anticoagulant et sans anticoagulant. Chaque tube a été identifié. Le sang collecté sur tube sec avait été utilisé, après décantation pour la récolte du sérum. Les sérums ont été recueillis dans des tubes et conservés dans une glacière qui avait été 
immédiatement acheminé au Laboratoire National d'Elevage à Ouagadougou pour le diagnostic sérologique de la Peste-Des Petits ruminants.

\subsection{Analyse des données}

3.4.1 Analyses de laboratoire: La technique modifiée de Mac-Master décrite par Ayres et Mara (Ayres et Mara, 1997) a été utilisée pour rechercher et identifier les œufs de Nématodes, Cestodes et les oocystes de Coccidies. Les œufs de Trématodes ont été recherchés par la méthode de la sédimentation (Ayres et Mara, 1997)

Le nombre d'CEufs Par Gramme (OPG) de fèces a été évalué.

Les analyses hémoparasitologiques ont été effectuées par la technique de centrifugation en

\section{RÉSULTATS}

\subsection{Les résultats de l'enquête}

4.1.1 Caractéristiques socio-économique des éleveurs enquêtés: La majorité des éleveurs d'ovins était de l'ethnie Peulh (74\%), suivis des Mossis (24\%) et des Dogons (2\%), et n'avait pas été à l'école ordinaire $(80 \%)$. Le niveau d'éducation se résumait à l'éducation coranique $(44 \%)$. Les femmes ne représentaient que $12 \%$ des participants à l'enquête.

\subsubsection{Caractéristiques des troupeaux} d'ovins: Les résultats ont montré que les élevages enquêtés comportaient des ovins de race Djallonké, Bali-Bali et des métisses issues des deux races précédentes. Les caractéristiques tube à hématocrite et par la réalisation et observation de frottis sanguins.

La technique utilisée pour rechercher des anticorps anti-PPR a été le test ELISA (Enzyme Linked Immunosorbent Assay).

3.4.2 Analyse statistique des données : Les données d'enquêtes ont été soumises au logiciel statistique EPI INFO version 6.2. Les moyennes des données numériques ont été calculées utilisant la statistique descriptive, et les fréquences des données qualitatives ont été calculées. Les données de laboratoire ont été soumises au logiciel MINITAB version 16. La comparaison des taux de prévalences a été faite selon le test de Khi-Deux et la comparaison du nombre moyen d'OPG a été faite selon le test de Student à 95\% d'intervalle de confiance.

générales des troupeaux sont présentés dans le Tableau 1 qui indique que les races Djallonké $(41,8 \%)$ et les métisses (48,9\%) étaient les plus exploités $(\mathrm{P}<0,05)$. De plus, les femelles représentaient environ 70,7 \% de l'effectif total $(\mathrm{N}=1163)(\mathrm{P}<0,05)$ des troupeaux d'ovins enquêtés. L'effectif moyen d'ovins par élevage était de $23,0 \pm 3,5$ ovins composé de 10,0 0 3,0 jeunes (moins de 18 mois) et de 13,0 \pm 4,0 adultes (plus de 18 mois).

Les données sur le type de logements des animaux, ont montré que $68 \%$ d'éleveurs enquêtés ne disposaient pas de logements, $2 \%$ disposaient d'un bâtiment fermé et que $30 \%$ avaient des parcs ou enclos pour les ovins.

Tableau 1 : Quelques caractéristiques des élevages ovins enquêtés ( $N=1163$ têtes)

\begin{tabular}{l|l|l|l|l|l|l}
\hline & \multicolumn{2}{|l|}{ Race } & \multicolumn{2}{l}{ Sexe (\%) } & \multicolumn{2}{l}{ Age (\%) } \\
\hline & $\begin{array}{l}\text { Effectifs (moyenne } \\
\pm \text { écart-type) }\end{array}$ & $\begin{array}{l}\text { Proportion } \\
\text { Dialles }\end{array}$ & Mâlo & Femelles & $\begin{array}{l}\text { Jeunes } \\
(<18 \text { mois })\end{array}$ & $\begin{array}{l}\text { Adultes } \\
(>18 \text { mois })\end{array}$ \\
Djallonke & $486 \pm 63^{\mathrm{a}}$ & 41,8 & $24,6^{\mathrm{b}}$ & $75,3^{\mathrm{a}}$ & $42,2^{\mathrm{a}}$ & $578^{\mathrm{a}}$ \\
Bali-Bali & $108 \pm 29^{\mathrm{b}}$ & 9,3 & $29,6^{\mathrm{b}}$ & $70,4^{\mathrm{a}}$ & $55,5^{\mathrm{a}}$ & $44,4^{\mathrm{a}}$ \\
Métisses & $569 \pm 95^{\mathrm{a}}$ & 48,9 & $33,2^{\mathrm{b}}$ & $66,8^{\mathrm{a}}$ & $45,0^{\mathrm{a}}$ & $55,0^{\mathrm{a}}$ \\
\hline
\end{tabular}

Race : Sur la même colonne les moyennes portant des lettres différentes sont significativement différentes au seuil de 5\% Sexe et: Sur la même ligne les moyennes portant des lettres différentes sont significativement différentes au seuil de 5\% Age : Sur la même ligne les moyennes portant des lettres différentes sont significativement différentes au seuil de 5\% 


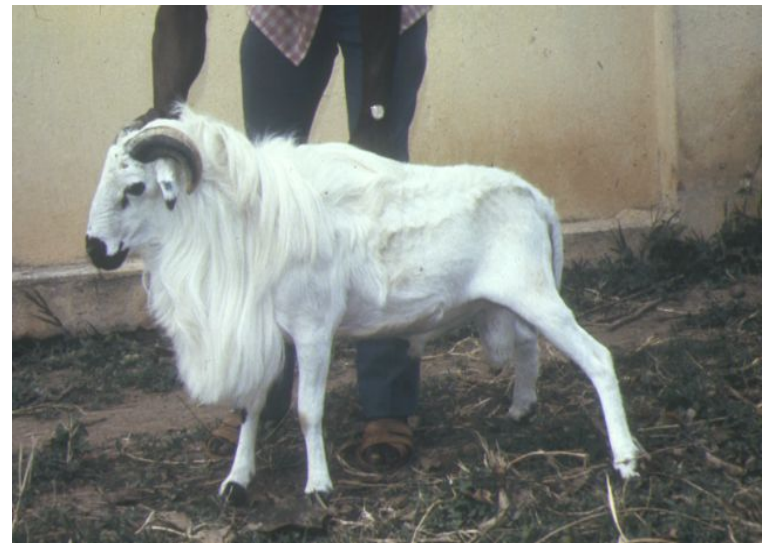

Figure 1 : mouton Djallonké mâle

\subsubsection{Connaissances des maladies et leur} traitement : Les diarrhées, les affections cutanées, et les avortements sont des symptômes de maladies les plus fréquemment

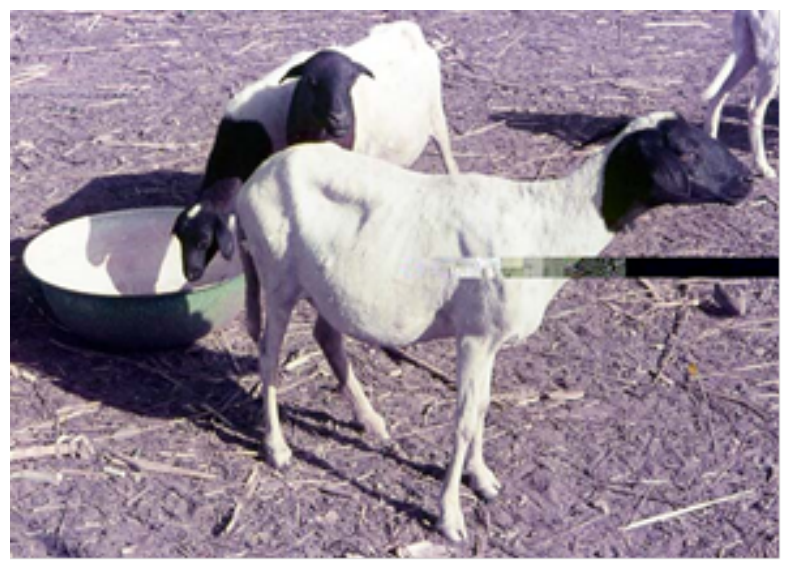

Figure 2 : Moutons métissés

cités par les éleveurs sur le plan sanitaire. La Figure 1 donne des informations sur la perception des éleveurs en matière de signes cliniques de maladies observées chez les ovins.

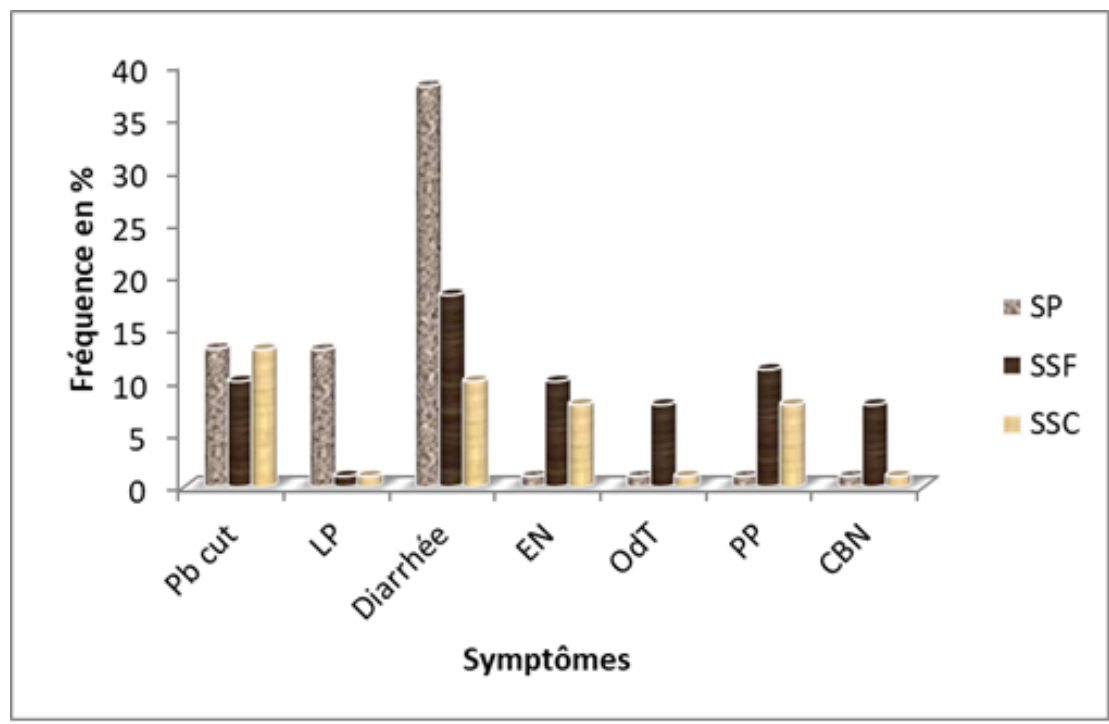

Figure 3: Fréquence des principaux symptômes de maladies suivant les saisons $\mathbf{P b} \mathbf{C u t}=$ Problèmes cutané, $\mathbf{L P}=$ lésions des pattes, $\mathbf{E N}=$ écoulement nasal, $\mathbf{O d T}=$ œdèmes de tête, $\mathbf{P P}=$ Poils piqués, $\mathbf{C B N}=$ croutes bucco nasales, $\mathbf{S P}=$ saison de pluies, $\mathbf{S S C}=$ Saison sèche chaude, $\mathbf{S S F}=$ Saison sèche froide.

4.1.4 Perceptions des éleveurs sur les facteurs responsables des maladies observées et traitement traditionnel des pathologies animales: Les tiques ont été reconnues comme étant responsables des problèmes cutanés et des lésions au niveau des pattes par 53,1\% des éleveurs. Les moustiques ont été indexés comme étant responsables des cas d'avortement chez les brebis par 9,38\%. Plus de la moitié $(56 \%)$ des éleveurs affirment vacciner leurs animaux contre la Pasteurellose et la Peste Des Petits Ruminants une fois par an et $20 \%$ utilisent des plantes médicinales pour soigner leurs animaux. Les différentes plantes utilisées sont regroupées dans le Tableau 2. Les éleveurs ont en outre signalé l'indisponibilité des produits vétérinaires $(23,3 \%)$ ainsi que des agents de santé sur le terrain $(23,3 \%)$. Pour l'amélioration de la santé du troupeau 34,5\% des ménages pensent qu'il faudrait améliorer 
l'alimentation en quantité et en qualité, d'autres $(24,3 \%)$ souhaitent avoir accès aux produits vétérinaires, certains $(16,1 \%)$ souhaitent une augmentation des agents vétérinaires sur le terrain tandis que 13,8 \% pensent qu'il faut aussi construire des abris pour les animaux.

Tableau 2: Traitements traditionnels des maladies ovines.

\begin{tabular}{|c|c|c|}
\hline Maladies & Produits & Méthode de traitement \\
\hline Diarrhée & Tapinathus sp & $\begin{array}{l}\text { Moudre les feuilles et rameaux, diluer dans l'eau et } \\
\text { faire boire }\end{array}$ \\
\hline Toux & Fedherbia albida & Faire boire aux animaux la décoction des écorces \\
\hline \multirow[t]{3}{*}{ Fièvre } & $-\quad$ ail & $\begin{array}{l}\text { Moudre les bulbes, diluer et mettre dans les fosses } \\
\text { nasales }\end{array}$ \\
\hline & - $\quad$ Guiera senegalensis & $\begin{array}{l}\text { Faire une décoction de feuilles mélangées au } \\
\text { tourteau de coton et faire boire aux animaux }\end{array}$ \\
\hline & Nicotiana tabacum & $\begin{array}{l}\text { Piler les feuilles, diluées dans l'eau et faire boire aux } \\
\text { ovins. }\end{array}$ \\
\hline
\end{tabular}

\subsection{Résultats des examens de} laboratoires

4.2.1 Le taux d'hématocrite: Le taux moyen d'hématocrite était de 26,0 $\pm 3,6 \%$. Les valeurs du taux d'hématocrite en fonction du sexe sont consignées dans le Tableau 3. Les valeurs moyennes étaient de 25,9 $\pm 3,58$ et de $25,7 \pm 4,20 \%$, respectivement pour les femelles et pour les mâles $(\mathrm{P}>0,05)$. L'hématocrite n'a pas présenté de différence significative en fonction de la classe d'âge. L'hématocrite moyen a été de $23,7 \pm 3,95 ; 27,2 \pm 3,75 ; 24,6$ $\pm 3,55$ et de $26,0 \pm 3,00$ respectivement pour les animaux des classes d'âge se situant entre 0-15 mois, 16-24 mois, 25-36 mois et supérieure à 36 mois (Tableau 4). Le Tableau 5 montre que la présence de parasites gastro-intestinaux n'a pas influencé significativement le taux d'hématocrite (P> 0,05). Les hématocrites moyens ont été de 24,2 \pm 4,00 et de 26,6 $\pm 3,30$ respectivement pour les animaux hébergeant ces parasites et ceux qui n'en hébergeant pas. Les valeurs d'hématocrite n'ont pas été significativement différentes entre les villages.

Tableau 3 : valeurs de l'hématocrite en fonction du sexe $(\mathrm{N}=150)$

\begin{tabular}{l|l|l|l}
\hline Ovins & Effectif & Hématocrite & Ecart-type \\
\hline Femelle & 105 & $25,9^{\mathrm{a}}$ & $\pm 3,58$ \\
Mâle & 45 & $25,7^{\mathrm{a}}$ & $\pm 4,20$ \\
\hline
\end{tabular}

${ }^{(a)}$ les valeurs de la même colonne suivies de lettres identiques ne sont pas statistiquement différentes au seuil de $5 \%(\mathrm{P}<0,05)$.

Tableau 4 : Hématocrite moyen en fonction de l'âge des animaux ( $N=150)$

\begin{tabular}{l|l|l|l}
\hline Age (mois) & Effectifs & Hématocrite & Ecart-type \\
\hline $0-15$ & 21 & $23,7 \mathrm{a}$ & 3,95 \\
$16-24$ & 60 & $27,2 \mathrm{a}$ & 3,75 \\
$25-36$ & 36 & $24,6 \mathrm{a}$ & 3,55 \\
$>36$ & 33 & $26,0 \mathrm{a}$ & 3,00 \\
\hline
\end{tabular}

$\left({ }^{\text {ab }}\right)$ Les valeurs de la même colonne suivies par des lettres différentes sont significativement différentes au seuil de $5 \%(\mathrm{P}<0,05)$ 
Tableau 5 : Hématocrite moyen en fonction de la présence ou de l'absence de parasites sanguinoles $(\mathrm{N}=150)$

\begin{tabular}{l|l|l|l}
\hline & Effectifs & Hématocrite moyen & Ecart type \\
\hline Echantillons négatif & 30 & 26,6 & $3,30^{\mathbf{a}}$ \\
Echantillons positif & 120 & 24,2 & $4,00^{\mathbf{a}}$ \\
\hline
\end{tabular}

$\left.{ }^{(a b}\right)$ Les valeurs de la même colonne suivies de lettres différentes sont significativement différentes au seuil de $5 \%(\mathrm{P}<0,05)$.

4.2.2 La coproscopie : La prévalence d'infestation parasitaire moyenne a révélé un taux d'infestation général de $86,7 \%$ et un nombre moyen d'OPG de $1149 \pm 706$ œufs. Les Nématodes (strongles), les Coccidies et les Cestodes (Monieza) ont été les principaux parasites rencontrés lors des examens parasitologiques. 38,5\% des échantillons ont présenté au moins deux parasites gastrointestinaux différents. Les prévalences moyennes générales étaient de $31,6 \%, 85,0 \%$ et $3,33 \%$, respectivement pour les Nématodes, les
Coccidies et les Cestodes $(\mathrm{P}<0,05)$. Les Tableaux 6 et 7 donnent des informations sur les prévalences et les nombre d'OPG observés pour chaque type de parasite. Les Coccidies ont présenté plus d'OPG comparativement aux Nématodes et aux Cestodes, ces derniers aussi ayant présenté plus d'OPG que les Nématodes $(\mathrm{P}<$ 0,05). Les degrés d'infestation correspondante étaient de 400 OPG pour les Monieza, 76,3 OPG pour les Strongles et de 1123 OPG pour les Coccidies (Tableau 7).

Tableau 6 : Prévalence d'infestation en fonction du type de parasite

\begin{tabular}{l|l|l}
\hline Type de parasite & Echantillons concernés & Prévalence $\mathbf{( \% )}$ \\
\hline Nématodes (strongles) & $48(\mathrm{~N}=150)$ & $31,6^{\mathrm{a}}$ \\
Coccidies & $130(\mathrm{~N}=150)$ & $86,0^{\mathrm{b}}$ \\
Cestodes (Monieza) & $5(\mathrm{~N}=150)$ & $3,33^{\mathrm{c}}$ \\
\hline
\end{tabular}

Les valeurs de la même colonne suivies de lettres différentes sont significativement différentes au seuil de $5 \%(\mathrm{P}<0,05)$

Tableau 7 : Effet du type de parasite sur le nombre d'OPG

\begin{tabular}{l|l|l|l}
\hline Type de parasite & $\begin{array}{l}\text { Echantillons } \\
\text { concernés }\end{array}$ & OPG & Ecart-type \\
\hline Nématodes (strongles) & 48 & $76^{\mathrm{a}}$ & 34 \\
Coccidies & 130 & $1123^{\mathrm{b}}$ & 467 \\
Cestodes (Monieza) & 5 & $400^{\mathrm{c}}$ & 101 \\
\hline
\end{tabular}

OPG = œuf par gramme.

(ab) Les moyennes de la même colonne suivies par des lettres différentes sont significativement différentes au seuil de 5\% (P<0,05).

4.2.2.1 Effet du sexe : La prévalence des infestations était de 76,9\% chez les femelles et 23,0 \% chez les males (Tableau 8 ). Les résultats du Tableau 9 ont montré une influence significative $(\mathrm{P}<0,05)$ du sexe sur l'OPG moyen qui a été supérieur chez les mâles (2250 \pm 902) comparativement aux femelles (819 \pm 259). 
Tableau 8 : Effet du sexe sur le degré d'infestation parasitaire

\begin{tabular}{l|l|l}
\hline Sexe & Effectifs & Prévalence(\%) \\
\hline Femelle & 105 & $76,9^{\mathrm{a}}$ \\
Mâle & 45 & $23,1^{\mathrm{b}}$ \\
Total & 150 & 100 \\
\hline
\end{tabular}

$\left({ }^{\mathrm{ab}}\right)$ Les valeurs de la même colonne suivies par des lettres différentes sont significativement différentes au seuil de 5\% (P<0,05).

Tableau 9 : Effet du sexe sur le nombre d'OPG

\begin{tabular}{l|l|l|l|l}
\hline Sexe & Effectifs & Prévalence & OPG moyen & Ecart-type \\
\hline Femelle & 105 & $76,9^{\mathrm{a}}$ & 819 & 259 \\
Male & 45 & $23,0^{\mathrm{b}}$ & 2250 & 902 \\
\hline
\end{tabular}

${ }^{(a b)}$ Les valeurs de la même colonne suivies par des lettres différentes sont significativement différentes au seuil de $5 \%(\mathrm{P}<0,05)$.

4.2.2.2 Effet de la classe d'âge: Le degré d'infestation parasitaire a varié en fonction de l'âge des ovins. La prévalence était de $44,2 \%$ pour les âges compris entre16 et 24 mois et de $7,70 \%$ pour les âges inferieurs à 15 mois $(\mathrm{P}<$ 0,05). Le nombre d' OPG moyen des classes d'âge comprises entre 16 et 24 mois (1577 \pm 812) a été le plus élevé de tous les groupes d'âge analysés et la différence était significative comparativement à la classe d'âge comprise entre 25 et 36 mois ( $571 \pm 140$ ) et la classe d'âge supérieure à 36 mois $(977 \pm 123)(\mathrm{P}<$ $0,05)$. La classe d'âge inférieur à 15 mois a également présentée un nombre moyen d'OPG

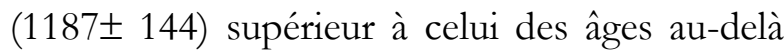
de 25 mois $(\mathrm{P}<0,05)$. Les données du degré d'infestations en fonction de l'âge sont présentées dans le Tableau 10 et celles du nombre d'OPG dans le tableau 11.

Tableau 10 : Effet de l'âge sur le degré d'infestation (prévalence)

\begin{tabular}{l|l|l}
\hline Classe d'âge (mois) & Effectifs & Prévalence $\%$ \\
\hline $0-15$ & 21,0 & $7,7^{\mathrm{a}}$ \\
$16-24$ & 60,0 & $44,2^{\mathrm{b}}$ \\
$25-36$ & 36,0 & $26,9^{\mathrm{b}}$ \\
$>36$ & 33,0 & $21,2^{\mathrm{b}}$ \\
TOTAL & 150 & 100 \\
\hline
\end{tabular}

$\left({ }^{\text {ab }}\right)$ Sur la même colonne les valeurs suivies par des lettres différentes sont significativement différentes au seuil de $5 \%(\mathrm{P}<0,05)$.

Tableau 11 : Effet de l'âge sur le nombre d'OPG

\begin{tabular}{l|l|l|l}
\hline Classe d'âge (mois) & Effectifs & OPG moyen & Ecart-type \\
\hline $0-15$ & 21 & $1187^{\mathrm{a}}$ & 144 \\
$16-24$ & 60 & $1577^{\mathrm{b}}$ & 812 \\
$25-36$ & 36 & $571^{\mathrm{c}}$ & 140 \\
$>36$ & 33 & $977^{\mathrm{d}}$ & 123 \\
\hline
\end{tabular}

${ }^{(a b)}$ les moyennes de la même colonne suivies par des lettres différentes sont significativement différentes au seuil de $5 \%(\mathrm{P}<0,05)$. 
4.2.2.3 Effet de la localité : La prévalence des infestations a été plus élevée dans le village de Nomon $(36,5 \%)$ contre 30,7 et $32,7 \%$ pour les villages de Nodin et Thiou ( $\mathrm{P}>0,05)$ (Tableau 12). Le nombre d'OPG moyen du village de
Nodin (700 \pm 181$)$ a été le plus faible comparativement aux valeurs observées à Thiou (1345 \pm 135$)$ et Nomon $(1352 \pm 122)$ ( $\mathrm{P}$ $<0,05)$ (Tableau 13).

Tableau 12 : Prévalence des infestations en fonction de la localité

\begin{tabular}{l|l|l|l|l|l|l}
\hline Localité & Effectifs & Prévalence \% & Infesté (+) & Non infesté (-) & Total & Valeur de P \\
\hline Nodin & 50 & $30,7 \mathrm{a}$ & 15 & 35 & 50 & - \\
Nomon & 50 & $36,5 \mathrm{a}$ & 18 & 32 & 50 & 0,5 \\
Thiou & 50 & $32,7 \mathrm{a}$ & 16 & 34 & 50 & 0,8 \\
Total & 150 & & 49 & 101 & 150 & \\
\hline
\end{tabular}

Tableau 13 : Effet de la localité sur le nombre d'OPG

\begin{tabular}{l|l|l|l}
\hline Localité & Effectifs & OPG moyen & Ecart-type \\
\hline Nodin & 50 & $700^{\mathrm{a}}$ & 181 \\
Nomon & 50 & $1352^{\mathrm{b}}$ & 122 \\
Thiou & 50 & $1345^{\mathrm{b}}$ & 135 \\
\hline
\end{tabular}

(a) les moyennes de la même colonne suivies par des lettres identiques ne sont pas significativement différentes au seuil de $5 \%(\mathrm{P}<0,05)$.

4.2.3 Sérologie: Une séroprévalence de $36,7 \%$ a été trouvé pour la Peste des Petits Ruminants (PPR) avec une variation en fonction du sexe, montrant 8,80\% chez les mâles et 43,8\% chez les femelles $(\mathrm{P}<0,05)$ (Tableau 14). Le Tableau 15 présente les résultats de la séroprévalence de la PPR en fonction de l'âge. Chez les ovins agés de plus de 25 mois, la prévalence a été significativement plus élevée $(45,5 \%) \quad(\mathrm{P}<0,05)$ comparativement aux classes d'age inferieurs à 25 mois. Les résultats en fonction de la localité ont montré une séroprévalence de la PPR élevée à Nodin (59,1\%), comparativement à Thiou $(22,7 \%)$ et Nomon $(18,2 \%)(\mathrm{P}<0,05)$, ces deux derniers n'ont pas présenté de différence significative (figure 4).

Tableau 14 : Effet du sexe sur la séroprévalence de la Peste des Petits Ruminants chez les ovins

\begin{tabular}{l|l|l|l|l|l|l}
\hline Sexe & Effectif & $\begin{array}{l}\text { séroprévalence } \\
(\mathbf{\%})\end{array}$ & $\mathbf{P P R}+$ & $\mathbf{P P R}-$ & Total & Valeur de $\mathbf{P}$ \\
\hline Femelle & 105 & $43,8^{\mathrm{a}}$ & 46 & 59 & 105 & - \\
Mâle & 45 & $8,8^{\mathrm{b}}$ & 4 & 41 & 45 & 0,00003 \\
Total & 150 & & 50 & 100 & 150 & \\
\hline
\end{tabular}

Tableau 15: Effet de l'âge sur la séroprévalence de la Peste des Petits Ruminants chez les ovins

\begin{tabular}{l|l|l|l|l|l|l}
\hline $\begin{array}{l}\text { Classe d'âge } \\
\text { (mois) }\end{array}$ & Effectif & $\begin{array}{l}\text { Prévalence } \\
\mathbf{0}\end{array}$ & PPR+ & PPR- & Total & Valeur de P \\
\hline $0-15$ & 21 & $4,55^{\mathrm{a}}$ & 1 & 20 & 21 & - \\
$16-24$ & 60 & $13,6^{\mathrm{a}}$ & 8 & 52 & 60 & 0,5 \\
$25-36$ & 36 & $36,4^{\mathrm{b}}$ & 13 & 23 & 36 & 0,02 \\
$>36$ & 33 & $45,5^{\mathrm{b}}$ & 15 & 18 & 33 & 0,004 \\
Total & 150 & & 37 & 113 & 150 & \\
\hline
\end{tabular}




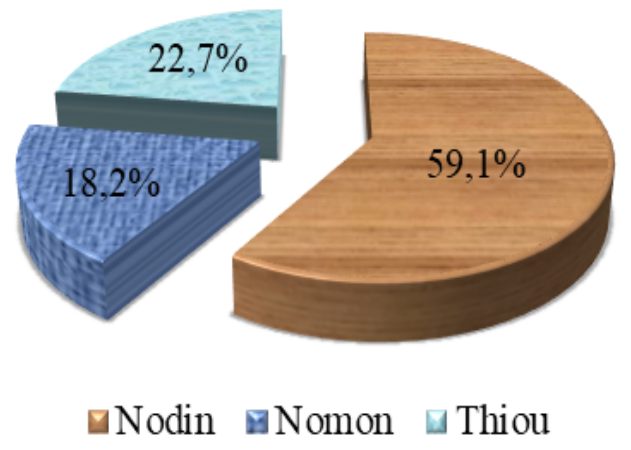

Figure 4 : Séroprévalence de la peste des Petits Ruminants selon le village

\section{$5 \quad$ DISCUSSION}

\subsection{Les données d'enquête}

5.1.1 Caractéristiques socio-économique des éleveurs enquêtés: Le faible niveau d'instruction et d'alphabétisation des éleveurs enquêtés observé dans cette étude avait été souligné par d'autres auteurs, et cette situation constitue une contrainte majeure en particulier pour la vulgarisation et le transfert des technologies aux éleveurs (MRA, 2010 ; Aruwayo et al., 2015). La proportion élevée de l'ethnie Peulh (74\%) et la faible proportion des femmes parmi les éleveurs d'ovins enquêtés sont également conformes aux données d'autres études au Burkina en particulier (Zonon, 2018) et en Afrique en général ou l'ethnie Peulh est reconnue pour leurs activités d'élevage des ruminants domestiques depuis les périodes ancestrales, et de ce fait le nom de cette ethnie a été donné à plusieurs races de ruminants dans les zones Sahéliennes (Doutressole, 1947 ; Epstein, 1971 ; Lebroy, 2003).

5.1.2 La structure du troupeau: La présence d'ovins de race Djallonké dans cette partie du Burkina (nord) serait liée à l'adaptation de cette race originaire des zones humides aux conditions agro-climatiques sahéliennes d'une part, et d'autre part, au choix porté par les éleveurs sur cette race ovines dont la rusticité et les performances zootechniques sont bien connues (Haye et al., 2004 ). La proportion élevée de femelles au sein des troupeaux serait en relation avec le caractère «Naisseur» des élevages extensif en Afrique conduits par l'ethnie Peulh pour qui les objectifs d'élevage sont généralement basés sur l'augmentation de la taille de l'effectif.

\subsubsection{Les perceptions sur les pathologies :} De nombreux signes cliniques de maladies avaient étés décrits par les éleveurs avec des fréquences plus élevées en saison de pluies. Ces données corroborent les trouvailles d'autres études réalisées dans ce domaine. En effet, il avait été démontré que la malnutrition et les mauvaises conditions d'hygiène rendaient les animaux moins résistant au parasitisme, au stress climatique (forte humidité) ainsi qu'aux diverses infections (Yusuf et al., 2018; Dagnachew et al., 2011). Ce constat implique que les risques d'exposition aux maladies soient plus élevés et que des interventions dans le domaine de la santé seraient aussi importantes en saison des pluies. La présente étude a également montré la connaissance empirique des éleveurs Peulh en matière de diagnostic et de traitement des maladies, et cela confirme l'assertion de BÂ qui stipulait que les Peulhs possèdent dans le domaine de la détection de la pathologie animale, une expérience certaine (BÂ, 1994). Les problèmes cutanés, les lésions des pattes et l'avortement décrits par les éleveurs dans cette étude avaient également été décrits comme symptômes majeurs en saison pluvieuse dans les élevages ovins au Cameroun (Miran and Makundi, 2017). La fréquence élevée de la diarrhée chez les animaux concorde avec les observations de Tchouamo (Tchouamo 
et al., 2005) qui a rapporté les diarrhées comme étant des problèmes majeurs de l'élevage ovin. L'utilisation de plantes médicinales dans le traitement des maladies animales signalée dans cette étude est une pratique très courante chez les éleveurs africains en général (Dassou et al., 2014). Ainsi, les Touaregs nigériens utilisent les racines de Calotropis procera pour soigner la gale des animaux, et les gousses d'Acacia arabica pour lutter contre le piétin (Bernus, 1969). Les graines d'Acacia nilotica et les feuilles d'Azadirachta indica ont été utilisées par les éleveurs du Yatenga (même zone d'étude) pour soigner la diarrhée des ovins (Boly et al., 2001). Cependant, au-delà de ces traitements traditionnels, la lutte contre les maladies animales devra être orientée vers la médecine vétérinaire moderne en se référant à un agent de santé. En effet, Face à certaines maladies, les éleveurs pratiquent de l'automédication et la majorité des soins apportés sont des antibiotiques, en injection ou en capsule avec très souvent les mêmes produits utilisés pour soigner diverses maladies.

\subsection{Les données de laboratoire}

\subsubsection{Hématologie}

5.2.1.1 Hématocrite : L'hématocrite moyen a été de 26,0 $\pm 3,6 \%$ chez les ovins. Ces valeurs sont proches de celles trouvées à l'ouest du Burkina Faso, soit 25,5 \pm 5,1 \% (Ye, 2012). La tendance s'expliquerait par la présence des mêmes races ovines, mais aussi de la similitude de conduite de ces animaux à l'ouest et au nord (site d'étude) du Burkina Faso. Cependant les valeurs sont inférieures à celles trouvées au Sénégal où une valeur d'hématocrite moyenne de 38\% avait été trouvée chez les ovins (Gueye et al., 1989). Le taux d'hématocrite faible dans la présente étude serait probablement lié au mode de conduite permanente des troupeaux au pâturage, car il a été démontré que des ovins conduits en permanence au pâturage avaient un hématocrite plus bas par rapport aux ovins en claustration permanente (Funkeu et al., 2000). L'infestation parasitaire pourrait aussi être à l'origine du faible taux d'hématocrite observé dans le cas présent, et cela avait été démontré par des auteurs (N’Dao et al., 1995 ; Oka et al 1999 ; Bastiaensen et al., 2003 ; Tanenbe et al., 2010). Le sexe des animaux n'a pas eu d'effet sur l'hématocrite, et ce constat avait été fait par Funkeu et al., (2000) au Cameroun. L'effet de l'âge n'a pas été significatif sur l'hématocrite, mais, l'hématocrite était légèrement plus bas chez les sujets âgés de moins de 24 mois. Ce même résultat avait été trouvé dans la même région par Ye en 2012, mais les présents résultats sont en désaccord avec des études au Nigeria qui ont révélé un effet significatif aussi bien de l'âge que du sexe sur le taux d'hématocrite chez les ovins (Egbe-Nwiyi et al., 2000). La localité n’a pas influencé significativement la valeur d'hématocrite. L'existence d'un même système de conduite des animaux dans ces villages pourrait expliquer cette tendance. La présence de parasites gastrointestinaux n'a pas eu d'effet significatif sur le taux d'hématocrite mais des valeurs plus faibles ont été observées chez les sujets parasités, et ces résultats concordent avec les observations faites à l'ouest du Burkina Faso sur des ovins (Ye, 2012).

5.2.1.2 Sérologie: La Peste Des Petits Ruminants continue toujours de faire des ravages dans les élevages ovins en Afrique (Couacy-Hymann, 2007). La prévalence globale de la Peste des petits ruminants (PPR) a été de $36,7 \%$ pour l'ensemble des sérums analysés. Cette prévalence est comparable à celle obtenues par Sow et al., chez les ovins dans la région du Soum (Sahel burkinabè) qui est une région voisine, soit 33,1 \% (Sow et al., 2008). La prévalence a été significativement plus élevée chez les animaux de plus de 36 mois (45,4\%) que chez les animaux de moins de 15 mois d'âge $(4,55 \%)$, et cela implique que les anticorps anti PPR augmentent significativement avec l'âge, et l'immunisation semble s'acquérir de manière significative après l'âge de 24 mois. Cela concorde avec les conclusions d'une étude au Burkina (Sow et al., 2008), et au Mali (Kamissoko et al., 2013). Une variation significative de la prévalence sérologique de la PPR en fonction de la localité a été observeé, avec le village de Nodin 
présentant la valeur la plus élevée (59,1\%) que Thiou $(22,7 \%)$ et Nomon $(18,2 \%)(\mathrm{P}<0,05)$. Cette différence pourrait s'expliquer à partir de la différence de classe d'âge des animaux des différentes localités qui ont probablement pu influencer la prévalence de la PPR comme expliqué ci-dessus, ou à la présence possible d'anticorps vaccinaux au niveau des prélèvements de Nodin.

5.2.2 Coprologie : Les méthodes d'analyse par flottaison et par sédimentation ont permis d'identifier plusieurs parasites gastro-intestinaux chez les ovins, incluant les Strongles $(31,6 \%)$, et les Monieza $(3,33 \%)$ et cela est en conformité avec d'autres études au Burkina et en Afrique (Belem et al., 2000; Wesongah et al., 2005; Opara et al., 2005 ; Ye, 2012) chez les ovins. Les taux de prévalence obtenus sont comparables aux valeurs trouvées par d'autres études à l'Ouest du pays, ou des prévalences de 2,38\%, $19,3 \%$ et $56,2 \%$ ont été observée, respectivement pour les Cestodes du genre Monieza, les Coccidies et les Strongles (Ye, 2012). La prévalence des Strongles (31,6\%) est nettement inférieure à celle trouvée au centre du Burkina Faso (Belem et al., 2000). La prévalence générale a été de $86,7 \%$, et cette prévalence reste supérieure à celles trouvées à l'Ouest du pays chez des ovins (65,45\%) (Ye, 2012). L'effet du sexe observé est en accord avec des auteurs qui ont aussi montré que le taux de prévalence des infestations parasitaires était plus élevé chez les femelles comparativement aux males (Dagnachew et al., 2011). Le degré d’infestation général était de

\section{CONCLUSION}

Cette étude a permis d'appréhender la problématique de la santé des ovins dans la commune rurale de Thiou à travers l'enquête transversale rétrospective et les études parasitologies et sérologiques. Les diarrhées, les lésions au niveau des membres et la fièvre ont été les symptômes majoritairement cités dans les élevages enquêtés. La lutte contre ces maladies en milieu rural repose sur la médecine vétérinaire «illicite» et la pharmacopée traditionnelle. Les analyses des prélèvements de
$1149 \pm 146$ OPG et l'effet du sexe a montré que les mâles excrétaient significativement plus d'œufs de nématodes que les brebis, et cela avait été trouvé également en Côte d'Ivoire (Achi et al., 2003). Le nombre d'OPG était plus élevé chez les jeunes (moins de 24 mois) comparativement aux sujets adultes, ce qui confirment l'assertion selon laquelle les jeunes sont plus sensibles aux pathologies que les adultes (Oka et al., 1999; Dagnachew et al., 2011). Le nombre d'OPG faible chez les sujets âgés pourrait s'expliquer par le fait que le contact permanent entre animaux et pâturages permet aux animaux de développer au fil des années, une certaine immunité qui va se caractériser par une tolérance à l'infestation parasitaire et une baisse du niveau d'expulsion des œufs parasitaires dans les fèces de ces animaux âgés (Zinsstag, 2000). Le degré d'infestation n'a pas varié significativement selon le village; toutefois des intensités parasitaires significativement plus basse ont été noté dans le village de Nodin comparativement aux villages de Thiou et Nomon ou les anticorps anti PPR étaient plus bas, et cela permet de supposer que les éleveurs de Thiou et Nomon n'avaient pas traité leurs animaux récemment avant les enquêtes, ce qui expliquerait le nombre élevé d'OPG (1345 OPG), et que dans les villages de Nodin il y aurait probablement des anticorps vaccinaux anti PPR stipulant que la prophylaxie médicale avait pu être fait dans ce village avant les enquêtes.

matières fécales ont permis de déterminer la prévalence des helminthes (Strongles, Tænia,) et les Coccidies ainsi que leur degré d'infestation. Il en est ressorti que les Coccidies étaient les plus représentées suivies des Strongles, que la prévalence des infestations était plus élevée chez les femelles alors que le nombre d'OPG était plus élevé chez les mâles, et que la prévalence de l'infestation ainsi que le nombre d'OPG étaient très élevés pour les âges compris entre 16 et 24 mois. L'analyse des 
prélèvements sanguins a permis de déterminer l'hématocrite qui n'a présenté aucune variation en fonction du sexe de l'âge et de la localité, ainsi que la séroprévalence de la Peste Des Petits Ruminants qui était très élevé chez les femelles et qui augmentait en fonction de l'âge des animaux, et était très élevé dans le village de Nodin comparativement à Thiou et Nomon. Il

\section{REMERCIEMENTS}

Les auteurs adressent leurs sincères remerciements aux acteurs du projet

\section{RÉFÉRENCES}

Achi Y l, Zinsstag J, Yèo N, Dea V and Dorchies P: 2003. Épidémiologie des helminthoses des moutons et des chèvres dans la région des savanes du Nord de la Côte d'Ivoire. Revue d'Elevage et de Médecine Vétérinaire des Pays Tropicaux 154 (3): 179-188.

Aruwayo A, Tiri GD, Yahaya MA. and Akinyemi M : 2015. An Emperical Analysis of Ruminant Production In Dutsinma Local Government Area, Katsina State, Nigerian International Journal of Inovation Agriculture \& Biological Research 3 (2):34-40.

Ayres RM. and Mara DD: 1997. Analyse des eaux résiduaires en vue de leur recyclage en agriculture. Manuel des techniques de laboratoire en parasitologie et bactériologie. Department of Civil Engineering University of Leeds, Leeds, England. World Health Organization, Geneva, 1997.

BÂ A S: 1994. L'art et la pharmacopée traditionnelle en Afrique sahélienne. Revue Scientifique et Technique office International des Epizooties 13(2) : 373-396.

Bastiaensen P, Dorny P, Batawui K, Boukaya A, Napala A. and Hendrickx G: 2003. Parasitisme des petits ruminants dans la zone périurbaine de Sokodé, Togo. Revue d'Élevage et de Médecine vétérinaire des Pays tropicaux Volume 56 (1-2): 43-50.

Belem AMG, Nikiema Zl, Sawadogo 1. and Dorchies PH: 2000. Parasites gastro- a été conclu que les pathologies ovines en générale, et les Coccidies, les Helminthes et la Peste Des Petits Ruminants en particulier, constituent toujours des menaces chez les ovins en saison des pluies dans la commune rurale de Thiou, d'où la nécessite de mettre en place un paquet technologique incluant la lutte contre ces pathologies.

ISIAE/CORAF/APESS qui ont financé la présente étude de recherche.

intestinaux des moutons et risques d'infestation parasitaire des pâturages en saison pluvieuse dans la région centrale du Burkina Faso. Revue d'Elevage et de Médecine Vétérinaire des Pays Tropicaux 151 (5): 437-442.

Bernus E : 1969. Maladies humaines et animales chez les Touaregs sahéliens. Journal de la société des Africanistes 39 (1):111-138.

Boly H, Ilboudo J B, Ouedraogo M, Berti F, Lebailly P. and Leroy P: 2001. L'élevage du "mouton de case" : Aspects techniques, socio-économiques et perspectives d'amélioration au Yatenga (Burkina Faso). Biotechnology Agronomie Societé et Environnment 5 (4): 201-208.

Couacy-Hymann E, Bodjo S C, Tounkara K, Koffi Y M, Ohui A H, DanhoT. and Bronsvoort BM: 2017 Comparison of two competitive ELISAs for the detection of specific Peste-Des-PetitsRuminant antibodies in sheep and cattle populations. African Journal of Biotechnology 6:732-736.

Dagnachew S, Asmare A. and Wudu T: 2011 Epidemiology of gastrointestinal helminthiasis of small ruminants in selected sites of North Gondar zone, Northwest Ethiopia. Ethiopian Veterinary Journal 15 (2): 57-68.

Dassou HG, Ogni CA, Yedomonhan H, Adomou AC, Tossou M, Dougnon JT. and Akoegninou A: 2014 Diversité, 
usages vétérinaires et vulnérabilité des plantes médicinales au Nord-Bénin. International Journal of Biological and Chemical Sciences $8: 1$.

Doutressole G: 1947 L'élevage en Afrique Occidentale Française. Larousse; Paris.

Egbe-Nwiyi TN, Nwaosu, SC. and Salami H A: 2000 Haematological values of appararently healthy sheep and goats as influenced by age and sex In arid zone of Nigeria. African Journal of Biomedical Research 3: 109 - 115.

Epstein H:1971 The origin of the domestic animals of Africa. African Publishing Corporation: UI. 101 5th avenue; NYUSA.

Fontes J. and Guinko S : 1995 Carte de la végétation et l'occupation du sol au Burkina Faso. Centre National de Recherche de l'Université de Toulouse III. IRD. Faculté des Sciences et Techniques de l'Université de Ouagadougou. Ministère de la Coopération Française-Projet Campus.67 p.

Funkeu NR, Pandey VS, Sorny P. and Killangas: 2000 Etude épidémiologique des nématodes gastro-intestinaux chez les ovins en milieu urbain et périurbain à Maroua, extrême nord du Cameroun. Revue d'Elevage et de Médecine Vétérinaire des Pays Tropicaux 53 (1):17-22.

Gueye A, Mbengue M. and Diouf A : 1989 Tiques et hémoparasitoses du bétail au Sénégal dans La zone sudsoudanienne. Revue d'Élevage et de Médecine vétérinaire des Pays Tropicaux 42 (4): 517-528.

Haye A, M'betiegue C, Nazaire I G. and Tanon B : 2004 Evaluation de la qualité du sperme du bélier de race Djallonke en région de savane humide de Côte d'Ivoire. Agronomie Africaine 16 (3): 37 46.

Kabore A: 2009 Activité anthelminthique de deux plantes tropicales testées in vivo et in vitro sur les strongles gastrointestinaux des ovins de races Mossi du
Burkina Faso. Thèse de Doctorat en développement rural $167 \mathrm{p}$.

Kamissoko B, Sidibe CAK, Niang M, Samake K, Traore A, Diakite A, Sangare O, Diallo A.and Libeau G: 2013 Prévalence sérologique de la peste des petits ruminants des ovins et des caprins au Mali. Revue d'Elevage et de Médecine Vétérinaire des Pays Tropicaux 66 (1): 5-10.

Lebroy M : 2003 Les soins au bétail chez les Peuls: une tradition ancestrale. Bulletin de la Société française d'bistoire de la médecine et des sciences vétérinaires $2: 2$.

Marchal JY : 1983 Yatenga, Nord Haute-Volta : la dynamique d'un espace rural soudano- sahélien (Travaux et Documents $\mathrm{N}^{\circ}$ 167). ORSTOM, Paris.

Miran BM. and Makundi AE: 2017 Participatory Epidemiology of Coenurus Cerebralis Infection on Sheep and Goats kept by pastoralists in N'gorongoro District, Tanzania. Huria Journal 24: 3 .

MRA (Ministère des Ressources Animales): 2004 Stratégie de Développement Rural à l'horizon 2015 (SDR), 99p.

MRA (Ministère des Ressources Animales) 2010: Plan d'actions et Programme d'Investissements du Sous-secteur de l'Elevage (PAPISE) 2010-2015. 61p.

N'dao M, Belot J, Zinsstag J. and Pfister : 1995 Epidémiologie des helminthoses gastrointestinales des PR dans la zone sylvopastorale au Sénégal. Veterinary Research 26:132-139.

Oka KC, Zinsstag J, Pandey VS, Fofana F. and N'depo A: 1999 Epidémiologie des parasites des ovins de la Côte d'Ivoire. Revue d'Elevage et de Médecine Vétérinaire des Pays Tropicaux 52 (1): 39-46.

Opara MN, Nwaobasi JK. and Okoli IC : 2005 Présence des helminthes chez les petits ruminants en élevage traditionnel à Owerri dans le Sud-est du Nigeria. Bulletin of Animal Health and Production Journal 53 (4): 226-233.

Sow A, Ouattara L, Compaore Z, Doulkom BR, Pare M, Poda G. and Nyambre J: 
2008 Serologic prevalence of Peste des Petits Ruminants in Soum Province, North of Burkina Faso. Revue d'Elevage et de Médecine Véterinaire des Pays Tropicaux Volume 61: 5-9.

Tanenbe C, Gambo H, Musongong AG, Boris O. and Achukroi MD : 2010 Prévalence de la trypanosomose bovine dans les départements du Faro et Déo, et de la Via au Cameroun: bilan de vingt années de lutte contre les glossines. Revue d'Elevage et de Médecine Vétérinaire des Pays Tropicaux 63 (3): 53-55.

Tchouamo JR, Tchoumboue J. and Thibault L : 2005 Caractéristiques socioéconomiques et techniques de l'élevage de petits ruminants dans la province de l'ouest du Cameroun. Tropicultura 23 (4) : 201-211.

Wesongah JO, Chemuliti J, Wesonga FD, Wanjala K, Munga L, Ngare P. and Murilla GA: 2005. Prevalence of parasitic infections in small ruminants in a pastoral community in Narok district, Kenya. Bulletin of Animal Health and Production Journal 53 (4): 218-225.

Ye A, 2012 : Contribution à la connaissance des pathologies des petits ruminants dans trois communes du Houet (Dandé, Padema et Satiri). Mémoire de fin d'études. IDR/ UPB, Option Elevage. Burkina Faso. 63p.

Yusuf A, Aruwayo A. and Muhammad IR : 2018 Characterisation of Small Ruminant Production Systems in SemiArid Urban Areas of Northern Nigeria. Journal of applied Sciences and Environmental Management Volume 22 Issue 5: 725-729. Retrieved December $8^{\text {th }} \quad 2018$ from: https://www.ajol.info/index.php/jasem http://ww.bioline.org.br/ja

Zinsstag J: 2000 Gastrointestinal of N'Dama cattle in the Gambia: Effects on productivity and options for control. ITMA-PhD.Thesis $\mathrm{n}^{\circ} 11$, ANTWERP. 154p.
Zonon K: 2018 Rôle de l'élevage dans la vulgarisation et le conseil agricole dans le cadre de la sécurité alimentaire et nutritionnelle au Burkina Faso. Mémoire de fin d'étude d'ingénieur du développement rural, Option élevage. Institut du Développement Rural IDR, Université NAZI BONI, 66 pp. 